\title{
The rate of hip osteoarthritis in patients with proximal femoral fractures versus hip contusion
}

\author{
B. Robstad • F. Frihagen $\cdot$ L. Nordsletten
}

Received: 18 May 2010 /Accepted: 1 March 2011 /Published online: 6 April 2011

(C) The Author(s) 2011. This article is published with open access at Springerlink.com

\begin{abstract}
Summary We found no difference in the rate of radiological hip osteoarthritis in the injured hip when comparing 349 patients with proximal femoral fractures and 112 patients with hip contusion. There was, however, a tendency for more osteoarthritis in patients with trochanteric fractures than in patients with femoral neck fractures.

Introduction Osteoarthritis $(\mathrm{OA})$ and osteoporotic fractures are two age-related disorders associated with considerable morbidity. There is a clinical impression of an inverse relation between osteoarthritis and osteoporosis, and a protective effect of OA against osteoporotic fractures has been proposed.

Methods We performed a case-control study in 461 subjects. Cases $(n=349)$ were patients aged 50 years or above who sustained a proximal femoral fracture from November 2003 to October 2004, registered prospectively in the department's fracture register. Controls $(n=112)$ were patients aged 50 years or above with the diagnosis of hip contusion, recruited from the hospital's discharge register. Radiographic OA was scored according to Kellgren and Lawrence (K\&L), and minimal joint space (MJS) was measured in both hips when possible. A K\&L grade II or higher or an MJS less than $2.5 \mathrm{~mm}$ was defined as OA.

Results Both in the hip fracture group and in the contusion group mean, the MJS was $3.5 \mathrm{~mm}$ on the injured side $(p=0.79)$. In the fracture group, $31 / 250(12 \%)$ had MJS $<2.5 \mathrm{~mm}$ and $16 / 112(14 \%)$ in the contusion group $(p=0.18)$. In the fracture
\end{abstract}

\footnotetext{
B. Robstad $\cdot$ F. Frihagen $\cdot$ L. Nordsletten $(\bowtie)$

Orthopaedic Department,

Oslo University Hospital and University of Oslo,

Kirkevn 166,

0407 Oslo, Norway

e-mail: lars.nordsletten@medisin.uio.no
}

group, 40/250 (16\%) had a K\&L OA grade II or higher, and in the contusion group 20/112 (18\%) persons had a K\&L OA grade II or higher $(p=0.66)$. There was a tendency for a higher incidence of $\mathrm{OA}$ in patients with trochanteric fractures compared with patients with cervical fractures.

Conclusions We found no differences on the injured side in the rate of hip OA between hip fracture patients and hip contusion patients.

Keywords Osteoarthritis · Osteoporosis · Osteoporotic fractures $\cdot$ Proximal femoral fractures

\section{Introduction}

Osteoarthritis (OA) and osteoporosis (OP) are two common, age-related disorders that are associated with considerable morbidity. The relationship between OA and OP has been examined in both community studies and case series. Studies of adult twins have shown an association between birth weight and bone mineral density (BMD) [1]. The twin studies have also shown that lumbar degenerative disc disease is similar in many ways to OA with evidence that degenerative disc disease is associated with a higher BMD at the hip and lumbar spine [2]. Data from Finland have shown that persons with poor height gain during childhood have an increase in their risk of hip fracture several decades later [3]. It has been suggested that the presence of OA protects against osteoporosis-related fractures [4-7], and that there is an inverse relationship between the two conditions [8-11]. A higher BMD and a trend toward a reduced risk of femoral neck fractures in patients with severe radiographic OA have been reported, but a significantly reduced risk of osteoporotic fracture was not found [11]. On the other hand, there are studies that have 
demonstrated a coexistence of the two entities. One study [12] found that OA did not protect against generalized primary OP. Glowacki et al. found occult osteoporosis and hypovitaminosis D in women with advanced OA [13]. In the Chingford study [14], a similar increase in bone resorption was found in patients with progressive knee $\mathrm{OA}$ as in patients with OP. They measured the level of urinary N-terminal and C-terminal, type I collagen telopeptides, both validated markers of bone resorption. A lower bone mineral density has been observed in patients with trochanteric fractures than with cervical fractures [15], and OA may give a trend toward a reduced risk of femoral neck fractures compared to trochanteric femoral fractures $[4,5]$. $\mathrm{OP}$ is a silent disease until fracture occurs, while OA gives a gradual onset of symptoms. A possible way to study the relation between osteoporosis and osteoarthritis is to assess the presence of osteoarthritis in patients with an osteoporosis-related fracture, such as a hip fracture, and compare patients with a similar trauma, but who did not sustain a fracture. A study with hip contusion patients forming a control group has, to our knowledge, not been performed previously. We, therefore, wanted to assess differences in the rate of hip OA between hip fracture and hip contusion patients. We also wanted to evaluate cervical and trochanteric femoral fractures in association with OA.

\section{Materials and methods}

We performed a retrospective, case-control study on 461 patients, 349 hip fracture patients (cases) and 112 hip contusion patients (controls). Hip fracture patients admitted from November 2003 to October 2004 were registered prospectively in the hospital's fracture registry. Four hundred one hip fracture patients were identified. The exclusion criteria were patients aged $<50(n=31)$, patients with a fracture in bone with a malignant disease $(n=6)$, patients with incomplete or missing radiographs $(n=14)$ and high-energy trauma $(n=1)$. This left 349 fracture patients for further analysis. The fractured hip was classified on the postoperative radiograph. Femoral neck fracture patients operated with arthroplasty $(n=89)$ were thus not included on the injured side. Preoperative radiographs were not used because they generally were of poor quality, but mainly because an intracapsular hematoma and the displacement of the femoral head in femoral neck fractures could influence the classifications, especially the minimal joint space (MJS). For ten patients, we could not retrieve the anteroposterior (AP) radiographs of the pelvis postoperatively. This left 250 patients with available postoperative radiographs of the fractured hip. Ninety-six of these were femoral neck fractures and 154 were trochanteric fractures. Separate analyses between the fracture types were performed. All 349 patients had interpretable radiographs of the non-injured side. There were no statistical differences in the proportion of women or mean age between the patients with interpretable ipsilateral radiographs and the ones operated with arthroplasty. The control group was recruited from the hospital's administrative registry and consisted of patients aged $\geq 50$ years admitted to our department with the ICD 10 diagnosis "contusion of hip" (S70) from November 2001 to October 2004. During the period in question, Ullevaal University Hospital served as a community hospital for about 200,000 people in Oslo. The organisation of the health system made it mandatory for all patients with an acute condition in need of hospital admittance - such as a hip fracture or hip contusion - to be admitted to the community hospital they belonged to by place of residence. A hip contusion was defined as a hip injury without fracture necessitating hospitalization. A stay of at least $6 \mathrm{~h}$ was interpreted as admittance. One hundred seventy-six patients were registered with a hip contusion. Forty patients were excluded due to previous arthroplasty on the contused side and 14 because of a previous internal fixation after a hip fracture. A further ten were excluded due to missing radiographs. This left 112 patients for further analysis. One of these had no radiograph of the non-injured side, and one had a previous total hip arthroplasty due to osteoarthritis on the non-injured side. AP radiographs of the pelvis were classified according to the grading system of Kellgren and Lawrence (K\&L) [16]. K\&L is a semiquantitative system using the radiographic features of OA (joint space narrowing, the existence of osteophytes, sclerosis and cyst formation), grading the osteoarthritis from 0 (normal hip) to 4 (severe osteoarthritis). K\&L grade II or higher indicates OA. We also measured MJS, a quantitative grading system with a cut-off point of $2.5 \mathrm{~mm}$ or less as the definition of hip osteoarthritis [17-20]. The grading was done by one of the authors (BR). The primary end point was the comparison of the rate of OA on the injured side as defined by either MJS or K\&L between cases and controls.

\section{Statistics}

For comparisons between the groups, independent samples $t$ test, chi-squared test and one-way ANOVA tests were used when appropriate with the SPSS version 16.0. The differences between the groups were reported as relative risk for dichotomous variables and mean differences for continuous variables. A correlation between measurements were analysed using the kappa coefficient for dichotomous variables and intraclass correlation coefficient for minimal joint space. $P$ values less than 0.05 were considered significant. 


\section{Observer reliability}

Twenty randomly selected radiographs were assessed twice with more than 1 year between assessments to estimate intraobserver variation. The mean difference between the measurements in MJS was $0.01 \mathrm{~mm}$ (SD, 0.23) and the largest difference was $0.5 \mathrm{~mm}$. The intraclass correlation coefficient was 0.98 . For the diagnosis of OA based on MJS, there was only one diagnosis that changed from the first to the second reading (kappa, 0.78). Similarly, for the diagnosis of OA, only one K\&L diagnosis differed between the first and second reading (kappa, 0.84).

\section{Results}

The mean age was 80.1 years in both groups $(p=0.97)$. There were $253(72 \%)$ women among the cases and 80 (71\%) in the control group $(p=0.83)$. In the case group, there were 172 patients $(49 \%)$ with a trochanteric fracture and $177(51 \%)$ with a femoral neck fracture. When using both grading systems combined, 48/250 (19\%) patients with hip fractures and 21/112 (19\%) patients with hip contusions had OA at the injured side (Table $1, p=0.92)$. At the non-injured side, we found that $61 / 349$ (18\%) had OA in the patients with hip fractures compared to $8 / 110(7 \%)$ in the hip contusion group using both classifications combined (Table $1, p=0.01$ ). The same pattern was found using $\mathrm{K} \& \mathrm{~L}$ grading and MJS, separately (Table 1). In a subgroup analysis comparing the two fracture types, there was 14/96 $(15 \%)$ with $\mathrm{OA}$ in the femoral neck group and 34/154 $(22 \%)$ in the trochanteric group (Table 2, $p=0.14)$. Similar results were found on the non-injured side (Table 2). We also compared each fracture separately with the controls for the presence of $\mathrm{OA}$ and found on the injured side that there was no difference between cases and controls. Overall, OA for femoral neck fractures was 14/96 (15\%) and for controls
$21 / 112(19 \%)$. This gave a relative risk of OA of $0.78(95 \%$ CI, 0.42 to $1.44, p=0.42$ ) for the fracture group compared with the control group. Comparing the trochanteric fractures with a rate of OA of $34 / 154(22 \%)$ to the controls (19\%) gave a relative risk (RR) of OA of 1.18 (95\% CI, 0.72 to $1.92, p=0.51$ ). For the non-injured side for the cases with femoral neck fractures, the rate of $\mathrm{OA}$ was $26 / 177$ (15\%) compared to $8 / 110$ (7\%) for the controls, giving a RR of OA of 2.02 (95\% CI, 0.95 to $4.30, p=0.06)$, and for the trochanteric fractures the rate of OA was $35 / 172(20 \%)$ giving a RR for OA of $2.80(1.35$ to $5.80, p=0.003$ ) compared to the controls. The mean MJS was $0.1 \mathrm{~mm}$ smaller in the femoral neck fracture patients than controls $(95 \% \mathrm{CI},-0.34$ to $0.10 ; p=0.27)$, and for the trochanteric fracture patients, MJS was $0.3 \mathrm{~mm}$ narrower $(95 \% \mathrm{CI},-0.05$ to $-0.49 ; p=0.02)$ compared to the controls.

When comparing OA as defined by MJS and K\&L, the Pearson correlation coefficient was $r=0.67(p<0.01)$ on the injured side and $r=0.72(p<0.001)$ on the non-injured side. The Pearson correlation coefficient of the overall OA between the injured and non-injured side was $0.24(p<0.001)$. Six patients in the fracture group, all with trochanteric fractures, and five patients in the contusion group, had bilateral osteoarthritis. Three patients in the contusion group had osteoarthritis only on the non-injured side.

\section{Discussion}

In this study, we did not find a difference in the prevalence of OA on the injured side in patients with hip fractures compared to patients with hip contusion. Hence, we found no support for the theory that OA may protect against a hip fracture. The relative risk was close to 1 with narrow confidence intervals for all comparisons, and the difference in mean MJS was very close to 0 (Table 1).

Table 1 Osteoarthritis measured by MJS and/or K\&L in the hip fracture group compared with the hip contusion group

\begin{tabular}{|c|c|c|c|c|}
\hline & Cases (hip fracture patients) & Controls (hip contusion patients) & $\begin{array}{l}\text { Mean difference or RR } \\
\text { with } 95 \% \text { confidence interval }\end{array}$ & $p$ \\
\hline MJS $\leq 2.5 \mathrm{~mm}$ ipsilateral $(n, \%)$ & $31 / 250(12 \%)$ & $16 / 112(14 \%)$ & $0.87(0.50$ to 1.52$)$ & 0.62 \\
\hline $\begin{array}{l}\text { K\&L grade II or higher } \\
\text { ipsilateral }(n, \%)\end{array}$ & $40 / 250(16 \%)$ & $20 / 112(18 \%)$ & $0.90(0.55$ to 1.46$)$ & 0.66 \\
\hline Osteoarthritis ${ }^{\mathrm{a}}$ ipsilateral $(n, \%)$ & $48 / 250(19 \%)$ & $21 / 112(19 \%)$ & $1.02(0.65$ to 1.63$)$ & 0.92 \\
\hline MJS ipsilateral (mean, SD) & $3.54(0.99)$ & $3.51(1.00)$ & $0.03(-0.19$ to 0.25$)$ & 0.79 \\
\hline MJS $\leq 2.5 \mathrm{~mm}$ contralateral $(n, \%)$ & $42 / 349(12 \%)$ & $8 / 110(7 \%)$ & $1.66(0.80$ to 3.41$)$ & 0.16 \\
\hline $\begin{array}{l}\mathrm{K} \& \mathrm{~L} \text { grade II or higher contralateral } \\
(n, \%)\end{array}$ & $52 / 349(15 \%)$ & $8 / 110(7 \%)$ & $2.05(1.00$ to 4.18$)$ & 0.04 \\
\hline Osteoarthritis $^{\mathrm{a}}$ contralateral $(n, \%)$ & $61 / 349(18 \%)$ & $8 / 110(7 \%)$ & $2.40(1.19$ to 4.87$)$ & 0.01 \\
\hline MJS contralateral (mean, SD) & $3.55(0.95)$ & $3.74(0.87)$ & $-0.20(-0.39$ to 0.00$)$ & 0.06 \\
\hline
\end{tabular}

${ }^{\text {a }}$ Osteoarthritis is defined as either an MJS $\leq 2.5 \mathrm{~mm}$ or a K\&L grade II or higher or previous surgery for osteoarthritis (total hip replacement) 
Table 2 Osteoarthritis measured by MJS and/or K\&L in the case group comparing femoral neck fractures and trochanteric fractures

\begin{tabular}{|c|c|c|c|c|}
\hline & $\begin{array}{l}\text { Cases, femoral neck } \\
\text { fractures }\end{array}$ & $\begin{array}{l}\text { Cases, trochanteric } \\
\text { fractures }\end{array}$ & $\begin{array}{l}\text { Mean difference or RR with } 95 \% \\
\text { confidence interval }\end{array}$ & $p$ \\
\hline MJS $\leq 2.5 \mathrm{~mm}$ ipsilateral $(n, \%)$ & $8 / 96(8 \%)$ & $23 / 154(15 \%)$ & $0.56(0.26$ to 1.19$)$ & 0.12 \\
\hline $\mathrm{K} \& \mathrm{~L}$ grade II or higher ipsilateral $(n, \%)$ & $10 / 96(10 \%)$ & $30 / 154(20 \%)$ & $0.54(0.27$ to 1.04$)$ & 0.06 \\
\hline Osteoarthritis ${ }^{\mathrm{a}}$ ipsilateral $(n, \%)$ & $14 / 96(15 \%)$ & $34 / 154(22 \%)$ & $0.66(0.37$ to 1.17$)$ & 0.14 \\
\hline MJS ipsilateral (mean, SD) & $3.72(0.90)$ & $3.42(1.03)$ & $0.30(0.05$ to 0.55$)$ & 0.02 \\
\hline MJS $\leq 2.5$ contralateral, $\mathrm{mm}(n, \%)$ & $15 / 177(9 \%)$ & $27 / 172(16 \%)$ & $0.54(0.30$ to 0.98$)$ & 0.04 \\
\hline $\begin{array}{l}\text { K\&L grade II or higher } \\
\text { contralateral }(n, \%)\end{array}$ & $25 / 177(14 \%)$ & $27 / 172(16 \%)$ & $0.90(0.55$ to 1.49$)$ & 0.68 \\
\hline Osteoarthritis $\mathrm{s}^{\mathrm{a}}$ contralateral $(n, \%)$ & $26 / 177(15 \%)$ & $35 / 172(20 \%)$ & $0.72(0.46$ to 1.15$)$ & 0.16 \\
\hline MJS contralateral (mean, SD) & $3.62(0.97)$ & $3.47(0.91)$ & $0.14(-0.06$ to 0.34$)$ & 0.16 \\
\hline
\end{tabular}

${ }^{a}$ Osteoarthritis is defined as either an MJS $\leq 2.5 \mathrm{~mm}$ or a K\&L grade II or higher or previous surgery for osteoarthritis (total hip replacement)

The relationship between OA and osteoporotic proximal femoral fractures is of special relevance to the ageing population because both conditions are common and both increase with age. It is of particular interest to investigate $\mathrm{OA}$ in the hip because it is often the only affected joint, suggesting that local biomechanical risk factors are important [21]. In this model, the fracture group represent patients with osteoporotic fractures and the contusion group represents patients with less osteoporosis, as their hip did tolerate a fall without fracturing. A possible explanation for the lack of difference in the level of osteoarthritis on the injured side may be that there are factors working in opposition. An OA may lead to an increase in BMD as a result of increased subchondral bone formation with stiffer bone, leading to mechanical stress on cartilage during impact loading and development of subchondral sclerosis and osteophytes [14, 22]. The protective effect of this against fracture may be outweighed by the effect osteoarthritis has on the hip in reducing range of motion, especially rotation and abduction/adduction, proprioception and muscle strength $[6,23]$ and thus increasing both the risk of falling and the risk of a fracture if a fall occurs.

When comparing the non-injured side, we found more $\mathrm{OA}$ in the fracture patients than in the contusion patients. The difference found on the non-injured side was unexpected, and no studies have, to our knowledge, previously reported this. Earlier studies have only investigated the injured side [5]. The results for the non-injured side should be interpreted with caution, as it is a post hoc exploratory analysis. However, a higher proportion of OA on the noninjured side in fracture patients may point to an influence on fall mechanics due to a stiffer joint with changed proprioception leading to a higher risk of fracture. The number of patients is larger on the non-injured side as we included the patients receiving a hemiarthroplasty for the analysis of the contralateral, uninjured hip.
There was a tendency towards more OA on the injured side for trochanteric fractures than for femoral neck fractures with an MJS in the hips with femoral neck fractures of $3.72 \mathrm{~mm}$ compared to $3.42 \mathrm{~mm}$ in the trochanteric fractures and a tendency towards more OA according to $\mathrm{K} \& \mathrm{~L}$ in the trochanteric group (Table 2). This supports previous findings of less OA in patients with femoral neck fractures than in patients with trochanteric fractures and gives some support to claims that OA protects against femoral neck fractures, but may lead to a relative increase in trochanteric fractures $[5,6,15,24]$.

The retrospective nature of this study leads to potential weaknesses. A selection bias is a potential problem with case-control studies. However, the cases were from our prospective in-house fracture register, and the controls were all patients with the diagnosis "hip contusion" from the discharge register, and thus unselected. The patients were recruited from the community hospital area and should be representative of the general population. A strength of our study is the use of a control group. Patients with hip trauma admitted to the hospital even in the absence of a fracture are probably frail, as most patients who contuse their hip will be treated as outpatients. The ones requiring admission may have previous hip pathology, such as osteoarthritis, which may be painful when traumatized. This, however, does not seem to be the case in our patients. The age and sex distribution in the control group was similar to the case group, and the selection of frail patients as controls suits the hip fracture patients well. A large number of excluded patients in both the case and the control group is a potential source of bias, especially the 89 patients with femoral neck fractures that were excluded from the analysis because they were operated with an arthroplasty and not available for measurements of osteoarthritis postoperatively. The quality of the preoperative radiographs of the fracture patients was not good enough to allow a precise measurement of the MJS or K\&L classification. The rate of OA on the non- 
injured side, however, was similar in the patients receiving an arthroplasty, and we found no indication that they differed from the other fracture patients. Another limitation of the study was that neither the symptoms of hip OA nor the duration of symptoms were registered. Although a hip fracture is a typical "osteoporotic" fracture, as few as $40 \%$ may have osteoporosis [25]. The measurement of BMD in our patients could have further clarified the relationship between OA and OP. We have, however, used criteria for OA that are in widespread use and well validated. One investigator evaluated all radiographs, and a large number of hips were investigated. There was a good correlation between the two chosen types of diagnostic criteria of OA (MJS and K\&L). The intraobserver reliability was also good. We present multiple tests and subgroup analyses. We could have restricted the statistical analysis to the main point of the study, i.e. only comparing the injured side of the hip fracture patients as a whole, with the controls, but we thought that the results on fracture type and non-injured side were worth reporting.

In the present study, there was no difference in the level of $\mathrm{OA}$ in patients with a hip fracture and patients who were hospitalized for hip contusion, hence the claim that OA protects against sustaining a hip fracture could not be supported.

\section{Conflicts of interest None.}

Open Access This article is distributed under the terms of the Creative Commons Attribution Noncommercial License which permits any noncommercial use, distribution, and reproduction in any medium, provided the original author(s) and source are credited.

\section{References}

1. Antoniades L, MacGregor AJ, Andrew T, Spector TD (2003) Association of birth weight with osteoporosis and osteoarthritis in adult twins. Rheumatology 42:791-796

2. Livshits G, Ermakov S, Popham M, Macgregor AJ, Sambrook PN, Spector TD et al (2010) Evidence that bone mineral density plays a role in degenerative disc disease: the UK Twin Spine Study. Ann Rheum Dis 69(12):2102-2106

3. Cooper C, Eriksson JG, Forsen T, Osmond C, Tuomilehto J, Barker DJ (2001) Maternal height, childhood growth and risk of hip fracture in later life: a longitudinal study. Osteoporos Int 12:623-629

4. Dequeker J, Goris P, Uytterhoeven R (1983) Osteoporosis and osteoarthritis (osteoarthrosis). Anthropometric distinctions. JAMA 249:1448-1451

5. Foss MV, Byers PD (1972) Bone density, osteoarthrosis of the hip, and fracture of the upper end of the femur. Ann Rheum Dis 31:259-264

6. Dretakis EK, Steriopoulos KA, Kontakis GM, Giaourakis G, Economakis G, Dretakis KE (1998) Cervical hip fractures do not occur in arthrotic joints. A clinicoradiographic study of 256 patients. Acta Orthop Scand 69:384-386

7. Dequeker J, Aerssens J, Luyten FP (2003) Osteoarthritis and osteoporosis: clinical and research evidence of inverse relationship. Aging Clin Exp Res 15:426-439

8. Cumming RG, Klineberg RJ (1993) Epidemiological study of the relation between arthritis of the hip and hip fractures. Ann Rheum Dis $52: 707-710$

9. Dequeker J, Johnell O (1993) Osteoarthritis protects against femoral neck fracture: the MEDOS study experience. Bone 14 (Suppl 1):S51-S56

10. Verstraeten A, Van EH, Haghebaert G, Nijs J, Geusens P, Dequeker J (1991) Osteoarthrosis retards the development of osteoporosis. Observation of the coexistence of osteoarthrosis and osteoporosis. Clin Orthop Relat Res 264:169-177

11. Cooper C, Cook PL, Osmond C, Fisher L, Cawley MI (1991) Osteoarthritis of the hip and osteoporosis of the proximal femur. Ann Rheum Dis 50:540-542

12. Makinen TJ, Alm JJ, Laine H, Svedstrom E, Aro HT (2007) The incidence of osteopenia and osteoporosis in women with hip osteoarthritis scheduled for cementless total joint replacement. Bone 40:1041-1047

13. Glowacki J, Hurwitz S, Thornhill TS, Kelly M, Leboff MS (2003) Osteoporosis and vitamin-D deficiency among postmenopausal women with osteoarthritis undergoing total hip arthroplasty. J Bone Joint Surg Am 85-A:2371-2377

14. Bettica P, Cline G, Hart DJ, Meyer J, Spector TD (2002) Evidence for increased bone resorption in patients with progressive knee osteoarthritis: longitudinal results from the Chingford study. Arthritis Rheum 46:3178-3184

15. Wolf O, Strom H, Milbrink J, Larsson S, Mallmin H (2009) Differences in hip bone mineral density may explain the hip fracture pattern in osteoarthritic hips. Acta Orthop 80:308-313

16. Kellgren JH, Lawrence JS (1957) Radiological assessment of osteoarthrosis. Ann Rheum Dis 16:494-502

17. Reijman M, Hazes JM, Koes BW, Verhagen AP, Bierma-Zeinstra SM (2004) Validity, reliability, and applicability of seven definitions of hip osteoarthritis used in epidemiological studies: a systematic appraisal. Ann Rheum Dis 63:226-232

18. Ingvarsson T, Hagglund G, Lindberg H, Lohmander LS (2000) Assessment of primary hip osteoarthritis: comparison of radiographic methods using colon radiographs. Ann Rheum Dis 59:650-653

19. Ingvarsson T, Hagglund G, Lohmander LS (1999) Prevalence of hip osteoarthritis in Iceland. Ann Rheum Dis 58:201-207

20. Lanyon P, Muir K, Doherty S, Doherty M (2003) Age and sex differences in hip joint space amongasymptomatic subjects without structural change: implications for epidemiologic studies. Arthritis Rheum 48:1041-1046

21. Birrell F, Croft P, Cooper C, Hosie G, Macfarlane GJ, Silman A (2000) Radiographic change is common in new presenters in primary care with hip pain. PCR Hip Study Group. Rheumatol (Oxf) 39:772-775

22. Naganathan V, Zochling J, March L, Sambrook PN (2002) Peak bone mass is increased in the hip indaughters of women with osteoarthritis. Bone 30:287-292

23. Stewart A, Black AJ (2000) Bone mineral density in osteoarthritis. Curr Opin Rheumatol 12:464-467

24. Meta M, Lu Y, Keyak JH, Lang T (2006) Young-elderly differences in bone density, geometry and strength indices depend on proximal femur sub-region: a cross sectional study in Caucasian-American women. Bone 39:152-158

25. Lyles KW, Colon-Emeric CS, Magaziner JS, Adachi JD, Pieper $\mathrm{CF}$, Mautalen C et al (2007) Zoledronic acid and clinical fractures and mortality after hip fracture. N Engl J Med 357:1799-1809 